\title{
Molecular docking studies of Calotropis gigantea phytoconstituents against Staphylococcus aureus tyrosyl-tRNA synthetase protein
}

\begin{abstract}
The current study reports Calotropis gigantea (C. gigantea) commonly known as milk weed contains phytoconstituents that have various medicinal properties. In Present study, Molecular docking analysis was also carried out to validate the anti-bacterial potential of these phytoconstituents. The reported previous studies of $C$. gigantea essential oil extract showed the GC-MS analysis and its screening found occurrence of nineteen compounds with different biological activity. The docking studies of $C$. gigantea phytoconstituents against $S$. aureus tyrosyl-tRNA synthetase (PDB ID: 1JIJ) found that it plays an essential role in protein synthesis by producing charged tRNAs. In docking analysis out of nineteen, there are seven compounds which shows highest docking score range -6.8 to -6.3 which are namely 2,4-dimethylacetophenone; 2-Methoxy-4-vinylguaiacol; 3-Phenyl-2-propenoic acid; 5-Methyl-2,4-diisopropylphenol; Eugenol; $\alpha$-Terpineol, L-Tyrosine Methyl ester. The docking analysis showed that all the nineteen compounds showed in range of -4.5 to $-6.8 \mathrm{Kcal} / \mathrm{mol}$ docking energy. The L-Tyrosine_Methyl_ester docking energy -6.7 , showing the maximum hydrogen bond interaction with TYR36, ASP40, ASP80 and GLN19. Thus, these studies might be beneficial for stretching information about an untouched site of this topic that will be helpful in declining this infection.
\end{abstract}

Volume 8 Issue 3 - 2020

\author{
Md Amjad Beg, Shagufta Ansari, Fareeda \\ Athar \\ Centre for Interdisciplinary Research in Basic Science, Jamia \\ Millia Islamia University, India
}

Correspondence: Fareeda Athar, Associate Professor, Centre for Interdisciplinary Research in Basic Sciences,

Jamia Millia Islamia University, New Delhi-I I0025, India,Tel +91II-2698I7I7 Ext. 4492, Fax +9|-II.26980I64 Email fathar@jmi.ac.in

Received: September 03, 2020 | Published: September 18, 2020

Keywords: Calotropis gigantea, Staphylococcus aureus, tyrosyl-tRNA synthetase, molecular docking

Abbreviations: C. gigantea, Calotropis gigantea; S. aureus, Staphylococcus aureus; TyrRS, tyrosyl-tRNA synthetase; RO5, Lipinski's rule of five; 3D, three-dimensional; RB, rotatable bond; HBA, hydrogen bond acceptor; HBD, hydrogen bond acceptor; log P, lipophilicity; TSPA, topological polar surface area

\section{Introduction}

Plants, which have at least one of its parts having substances that can be utilized for treatment of diseases, are called therapeutic plants. ${ }^{1}$ Drugs got from plants are generally acclaimed because of their wellbeing, simple accessibility and ease. Home grown meds may incorporate entire pieces of plant or generally set up from leaves, roots, bark, seed and blossoms of plants. Medicinal plants are the "spine" of customary medication, which implies more than 3.3 billion individuals in the less evolved nations. ${ }^{2,3} \mathrm{C}$. gigantea Linn is an enduring bush normally known as milkweed or wasteland weed. It has a place with family Asclepiadaceous and was assessed for its anticonvulsant movement. It has groups of waxy blossoms that are either white or lavender in shading. ${ }^{4}$ There is a constant need of the advancement of new compelling antimicrobial medications due to the development of new irresistible ailments and medication opposition. ${ }^{5,6}$ In Calotropis gigantea Linn. bloom constituent, for example, 2,3-Dihydro-benzofuran, 2,4-dimethylacetophenone, 2-Methoxy-4-vinylguaiacol, 3,7-Dimethyl-1,6-octadien-3-ol, 3,7-dimethyl-2,6-octadien-1-ol, 3-Phenyl-2-propenoic corrosive, 3-Thiophenemethanol, 5-Methyl-2,4-diisopropylphenol, 6-hepten1-ol, 2-Methyl, Benzaldehyde, Benzyl liquor, Cinnamyl liquor, Eugenol, Furfural, $\alpha$-Terpineol, L-Tyrosine Methyl ester, Phenethyl liquor, Pinocampheol, Trans-3-Hexen-1-ol. ${ }^{7}$ The plant is accounted for pain relieving action, antimicrobial movement, cell regeneration action, hostile to pyretic action, insecticidal action, cytotoxicity action, hepatoprotective action, pregnancy interceptive properties, laxative properties, pro coagulant action and wound mending action. ${ }^{8}$ ESKAPE is an abbreviation including the names of six bacterial microorganisms generally connected with antimicrobial opposition (Detection of ESKAPE Bacterial Pathogens at the Point of Care Using Isothermal DNA-Based Assays). ESKAPE is an abbreviation for their names and a reference to their capacity to get away from the impacts of generally utilized anti-infection agents through developmentally created systems. ${ }^{9-13}$ Therapeutic herbs are more noteworthy to the soundness of individual and network. ${ }^{14}$ In this manuscript author exhibit different viewpoints foreseeing job of these mixes in grip of the bacterium to the host cell and accordingly accommodating in forestalling the disease and diminishes mortality because of this ailment.

\section{Methodology}

\section{Physiochemical properties}

The physiochemical properties of the selected compounds were anticipated by openly accessible online SwissADME programming. Toxic capacity is imperative to assess in sedate planning as it help in deciding the harmful portion in animal model studies and decreases the quantity of animal model investigations. ${ }^{15,16}$

\section{Molecular docking: receptor and ligand preparation}

The 2D structures of Calotropis gigantea 20 phytoconstituents was downloaded by PubChem online server which is an openly accessible tool. The structures were then changed into. Mol2 design with the assistance of Chem3D Ultra, another bundle of Chem Office. ${ }^{17}$ 


\section{Target selection and preparation}

Prior detailed three-dimensional (3D) structure of the Crystal structure of $S$. aureus TyrRS in complex with SB-239629 (PDB ID: $1 \mathrm{JIJ})$ was recovered from RCSB PDB. ${ }^{18}$ The water particles just as cosolidified ligands were removed from the PDB document and include polar hydrogen bonds.

\section{Docking protocol}

Before we moving to the molecular docking, the topological investigation of the protein structures including dynamic binding site/ pocket was controlled by utilizing CASTp 3.0. ${ }^{19}$ Some residues are reasonable for anticipated ligand restricting site in the protein where the ligand can reversibly bind. Anyway other amino acid residues of the protein were giving right direction and affirmation. ${ }^{20}$ To decide the coupling mode and cooperation of the selected compounds and target, docking studies were performed by AutoDock/vina. ${ }^{21,22}$ The pdbqt files of the receptor protein, and $C$. gigantea compounds alongside the grid box getting at the dynamic site of the receptor for compound interaction was done through Auto Dock GUI program. The framework size limits along $\mathrm{X}, \mathrm{Y}$, and $\mathrm{Z}$ hub was scaled at 40 $\AA$ with a network dividing of $1 \AA$ to permit appropriate binding adaptability at the docked site. The yield pdbqt documents were composed into a design (conf) file. The receptor was dealt with unbending element while ligands were kept adaptable to achieve the best fitting compliance regarding the receptor complex. The created arrangements of docking were grouped and those with root mean square deviation (RMSD) esteem $<1.0 \AA$ were viewed as just referred in given reference. ${ }^{23-25}$ The coupling adaptation of ligands with the least binding affinity was portrayed as the steadiest compliance of the ligands as for the receptor.

\section{Protein-ligand interaction studies}

The interaction investigations of the protein-ligand complexed files perception were finished by PyMOL programming 1.3 variant.
PyMOL can create large 3D picture of small atoms and protein. The polar (hydrogen bond) and non-polar connections among receptor and little ligand(s) were pictured by PyMOL programming. For binding investigation of the $2 \mathrm{D}$ cooperation, this was done by Discovery Studio 2019 (BIOVIA). ${ }^{26-29}$

\section{Results}

\section{Calotropis gigantea flower extract phytoconstituent(s)}

The phytoconstituent of $C$. gigantea flower extracts (Figure 1). The pharmacokinetics examination was done by SwissADME server where the atomic weight was denoted as a noteworthy quality of the in therapeutic method of the medication activity. In C. gigantea, natural compounds were in the range $\leq 500$. Appropriately, RO5 the small atomic molecules of medications is transported, diffuse and ingested with no deterrent when contrasted with high subatomic weight. In compound examination RO5, subsequent stage is number of acknowledged hydrogen bond ( $\mathrm{O}$ and $\mathrm{N}$ particles) and number of acceptor hydrogen bond $(\mathrm{NH}$ and $\mathrm{OH})$ inside Lipinski's cut off points extend from 0-10 (H-bond acceptor) and 0-5 (H-bond contributor) separately. Lipophilicity (log P) and Topological Polar Surface Area (TPSA) values are critical properties for the gauge of oral risk of medication particles. The extending of $\log \mathrm{P}$ from (0-5) or the majority of the mixes run from 0.94-5.00 ( $\leq 5)$, which is as far as possible for medication to infiltrate bio-layer. The computation of the surface regions which is involved by oxygen, nitrogen and through appended hydrogen iota is called Topological Polar Surface Area (TPSA). Along these lines, the TPSA is carefully related to the hydrogen holding capability of the compound. C. gigantea flower phytoconstituents were found to have the best medication like properties. For a medication, a decent bioavailability is more probable for mixes with $\leq 10$ rotatable bonds and TPSA of $\leq 140 a$. All the TPSA, miLogP, number of particles, number of rotatable bonds and bioavailability (Table 1).

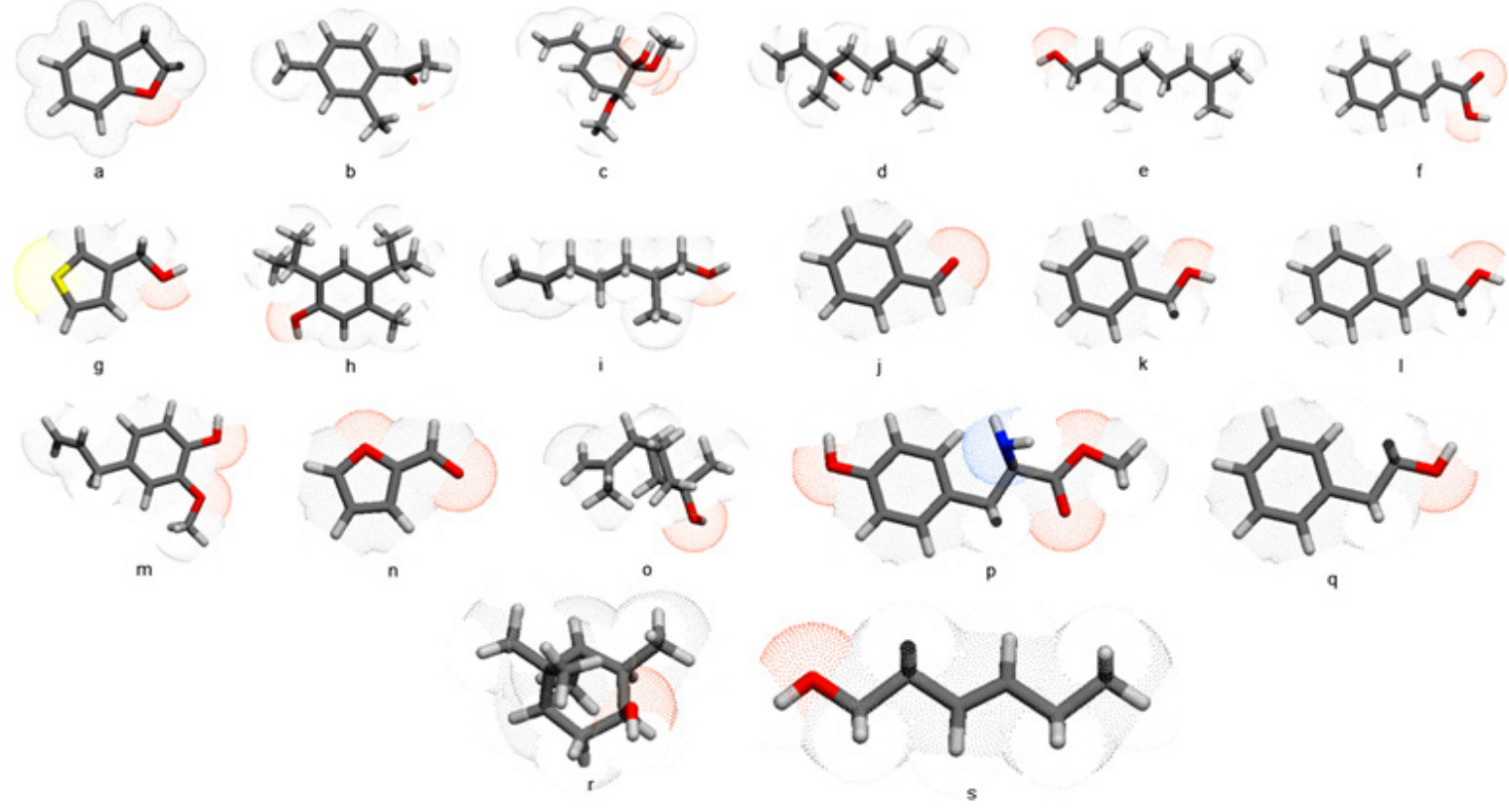

Figure I Phytoconstituent of $C$. gigantea flower extract (compound name enlisted in Table I). 
Table 1 Physiochemical parameters of $C$. gigantea flower extract phytoconstituents

\begin{tabular}{|c|c|c|c|c|c|c|c|c|}
\hline Compounds & Formula & MW & RB & HBA & HBD & MR & $\log P$ & RO5 \\
\hline 2,3-Dihydro-benzofuran & $\mathrm{C}_{8} \mathrm{H}_{8} \mathrm{O}$ & 120.15 & 0 & I & 0 & 35.79 & 1.89 & Y \\
\hline 2,4-dimethylacetophenone & $\mathrm{C}_{10} \mathrm{H}_{12} \mathrm{O}$ & 148.20 & I & 1 & 0 & 46.57 & 2.11 & Y \\
\hline 2-Methoxy-4-vinylguaiacol & $\mathrm{C}_{10} \mathrm{H}_{14} \mathrm{O}_{3}$ & 182.22 & 3 & I & 1 & 50.02 & 2.13 & Y \\
\hline 3,7-Dimethyl-I,6-octadien-3-ol & $\mathrm{C}_{10} \mathrm{H}_{18} \mathrm{O}$ & 154.25 & 4 & I & 1 & 50.44 & 2.71 & Y \\
\hline 3,7-dimethyl-2,6-octadien-I-ol & $\mathrm{C}_{10} \mathrm{H}_{18} \mathrm{O}$ & 154.25 & 4 & I & I & 50.40 & 2.75 & Y \\
\hline 3-Phenyl-2-propenoic acid & $\mathrm{C}_{9} \mathrm{H}_{10} \mathrm{~N}_{2} \mathrm{O}$ & 162.19 & 3 & 2 & 2 & 47.05 & 0.69 & Y \\
\hline 3-Thiophenemethanol & $\mathrm{C}_{5} \mathrm{H}_{6} \mathrm{OS}$ & 114.17 & I & I & 1 & 30.45 & 1.54 & Y \\
\hline 5-Methyl-2,4-diisopropylphenol & $\mathrm{C}_{13} \mathrm{H}_{20} \mathrm{O}$ & 192.30 & 2 & I & 1 & 62.59 & 2.86 & Y \\
\hline 6-hepten-I-ol, 2-MethyI & $\mathrm{C}_{8} \mathrm{H}_{16} \mathrm{O}$ & $|28.2|$ & 5 & I & 1 & 41.26 & 2.36 & Y \\
\hline Benzaldehyde & $\mathrm{C}_{7} \mathrm{H}_{6} \mathrm{O}$ & 106.12 & 1 & I & 0 & 31.83 & 1.36 & Y \\
\hline Benzyl alcohol & $\mathrm{C}_{7} \mathrm{H}_{8} \mathrm{O}$ & 108.14 & I & I & I & 32.57 & 1.66 & Y \\
\hline Cinnamyl alcohol & $\mathrm{C}_{9} \mathrm{H}_{10} \mathrm{O}$ & 134.18 & 2 & I & I & 42.50 & 1.98 & Y \\
\hline Eugenol & $\mathrm{C}_{10} \mathrm{H}_{12} \mathrm{O}_{2}$ & 164.20 & 3 & 2 & 1 & 49.06 & 2.37 & Y \\
\hline Furfural & $\mathrm{C}_{5} \mathrm{H}_{4} \mathrm{O}_{2}$ & 96.08 & I & 2 & 0 & 24.10 & 1.03 & Y \\
\hline$\alpha$-Terpineol & $\mathrm{C}_{10} \mathrm{H}_{18} \mathrm{O}$ & 154.25 & 1 & 1 & 1 & 48.80 & 2.09 & Y \\
\hline L-Tyrosine Methyl ester & $\mathrm{C}_{10} \mathrm{H}_{13} \mathrm{NO}_{2}$ & 195.22 & 4 & 4 & 2 & 51.84 & 1.39 & Y \\
\hline Phenethyl alcohol & $\mathrm{C}_{8} \mathrm{H}_{10} \mathrm{O}$ & 122.16 & 2 & 1 & 1 & 37.38 & 1.70 & Y \\
\hline Pinocampheol & $\mathrm{C}_{10} \mathrm{H}_{18} \mathrm{O}$ & 154.25 & 0 & I & I & 46.86 & 2.34 & Y \\
\hline Trans-3-Hexen-I-ol & $\mathrm{C}_{6} \mathrm{H}_{12} \mathrm{O}$ & 100.16 & 3 & I & I & 31.64 & 1.94 & Y \\
\hline
\end{tabular}

\section{Docking studies}

The prediction of the active binding site/pocket for $S$. aureus TyrRS (PDB ID: 1JIJ) was shown using CASTp 3.0 server (Figure 2). Molecular docking was done by AutoDock/vina ${ }^{21}$ using rigid docking method which was used because of the finding inhibitor and each step and binding must be examined. The grid box getting at the active site of the receptor for compounds binding was done through Auto Dock GUI program. Grid were generated, centre of the grid box X-11.179,
Y-11.702 and Z-91.461 else the dimensions of the grid box X-20, Y-22 and Z-20 for the prepared proteins. For S. aureus TyrRS, the grid was generated around the bound ligand SB-239629 (2S)-2-[[(2S)-2Amino-3-(4-hydroxyphenyl)propanoyl]amino]-2-[(2S,3S,4S,5S)1,3,4,5-tetrahydroxy-4-(hydroxymethyl)piperidin-2-yl]acetic acid (Table 2). The docked PDB ID: 1JIJ bound ligand SB-239629 had docking score $-7.5 \mathrm{kcal} / \mathrm{mol}$ and else the phytoconstituents of $C$. gigantea dock score against PDB ID: 1JIJ.
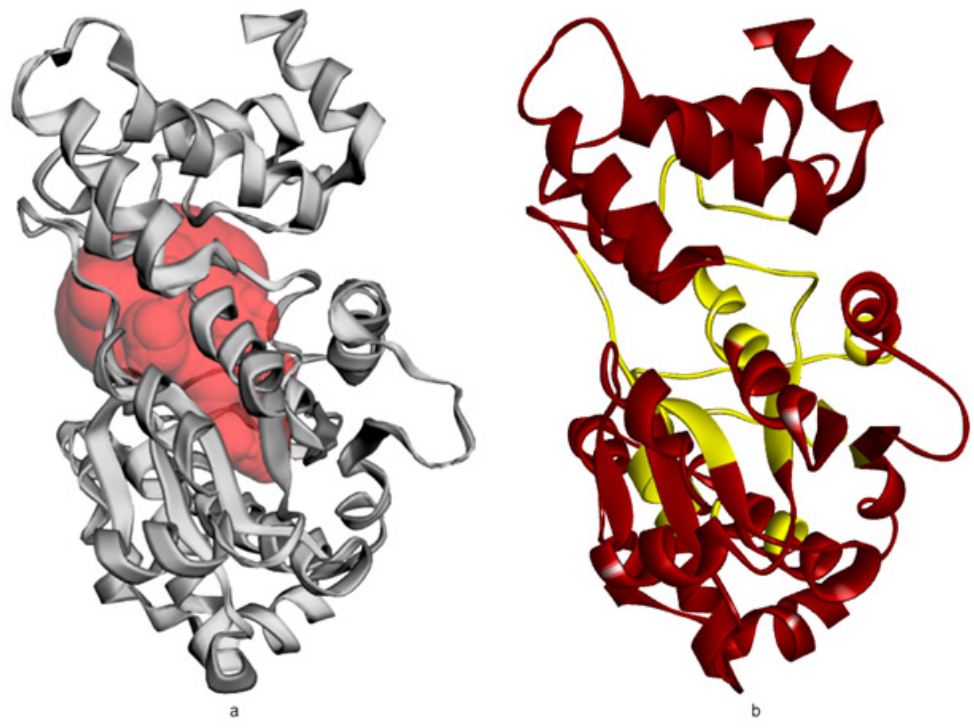

Figure 2 Cartoon representation of S. aureus TyrRS (A) the dot (red) representation in target protein showing active pocket by CASTp 3.0 (B) Crystal structure of S. aureus TyrRS (yellow) secondary structure visualize the region of amino acid involving active pocket by BIOVIA. 


\section{Protein-ligand complex interaction}

After docking analysis the protein-ligand complex file analysing the interacting protein binding sites (amino acid), the phytoconstituents of $C$. gigantea flower extract against $S$. aureus tyrosyl-tRNA synthetase proteins (PDB:1JIJ) reveals, all the ligands binding active sites (means amino acid residues) occupy active pockets of the proteins
(Figure 3). In these studies of ligand-receptor interactions depicts, $S$. aureus TyrRS target protein having highest interaction that is shown in (Figure 4). The ligand-receptor complex resolve the important conundrum for which residues are substantial for protein stabilization and otherwise important residue that are important for involved in protein conformation modification (Table 3).

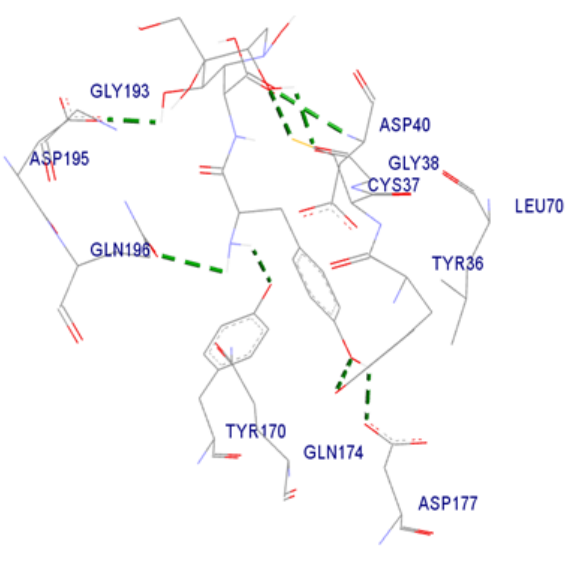

(A)

PDB ID: IJ_SB-239629.

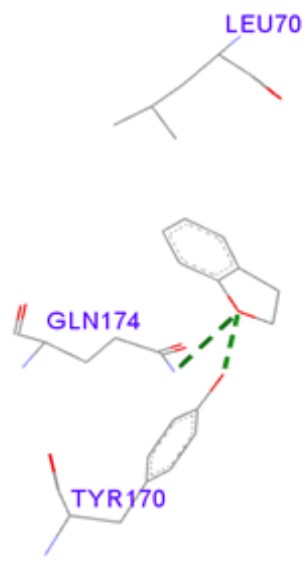

(A)

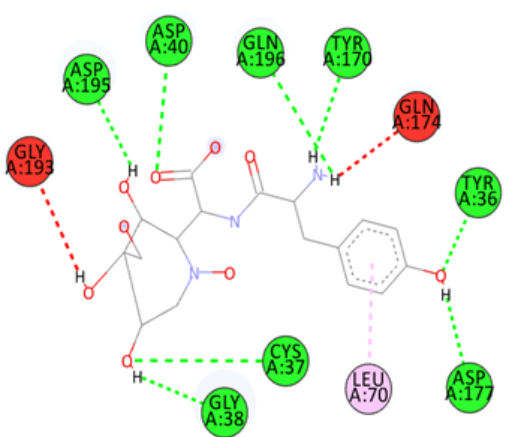

(B)

PDB ID: إI_2,3-Dihydro-benzofuran.

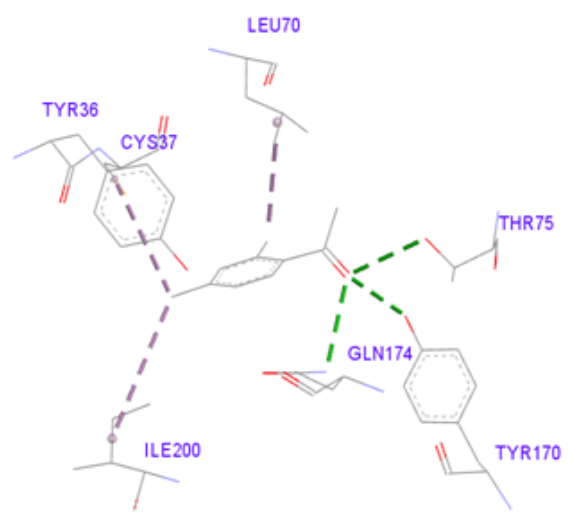

(A)

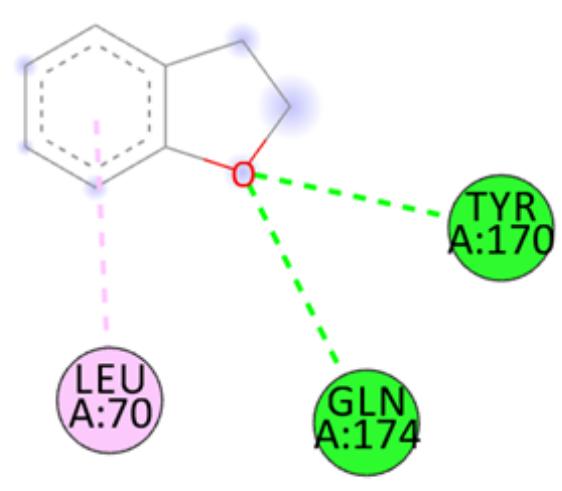

(B)

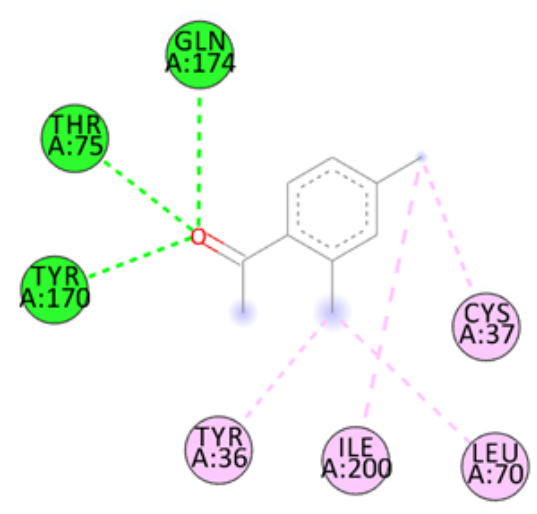

(B)

PDB ID: IJI_2,4-dimethylacetophenone2,4-dimethylacetophenone. 


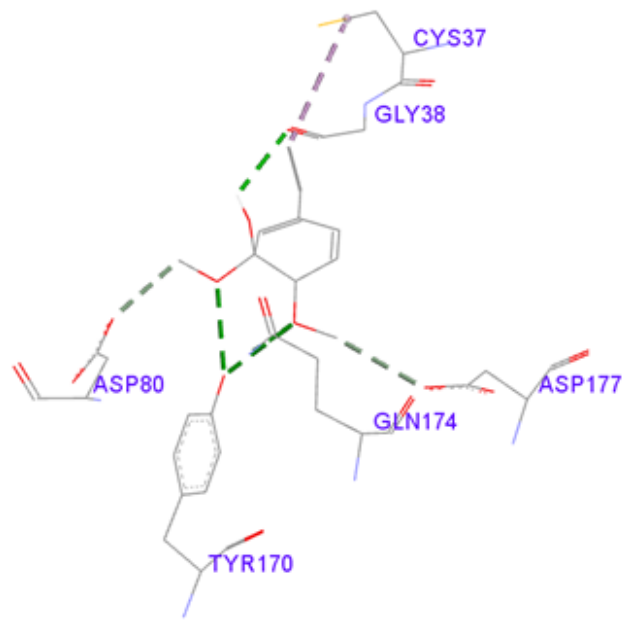

(A)

PDB ID: IJIJ_2-Methoxy-4-vinylguaiacol.

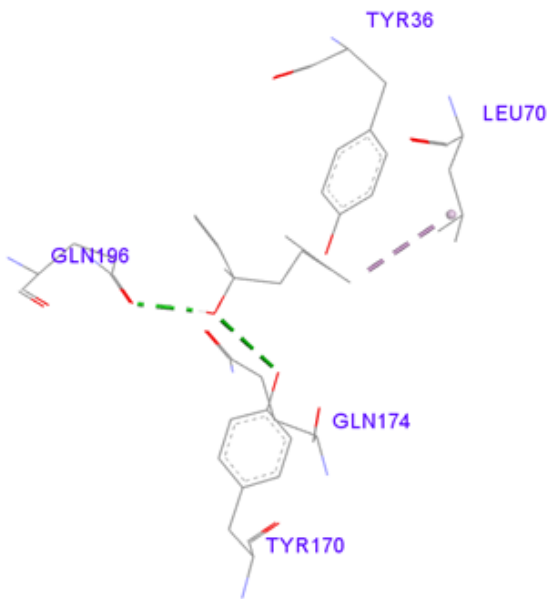

(A)

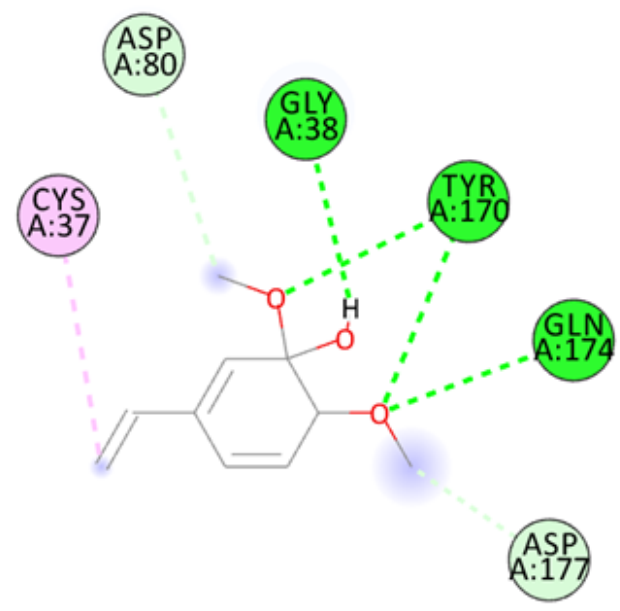

(B)

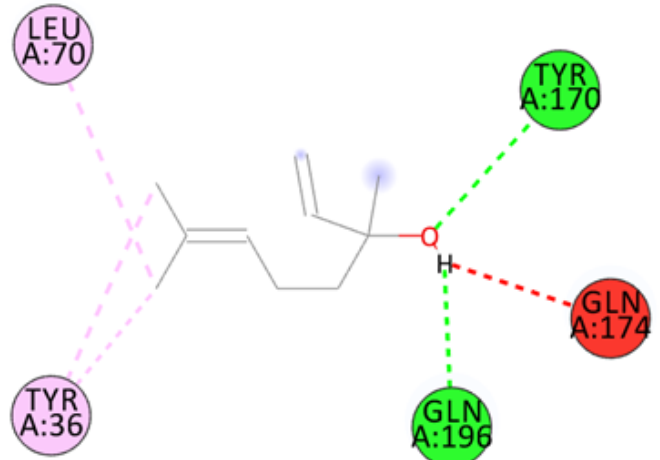

(B)

PDB ID: IJI_3,7-Dimethyl-I,6-octadien-3-ol.

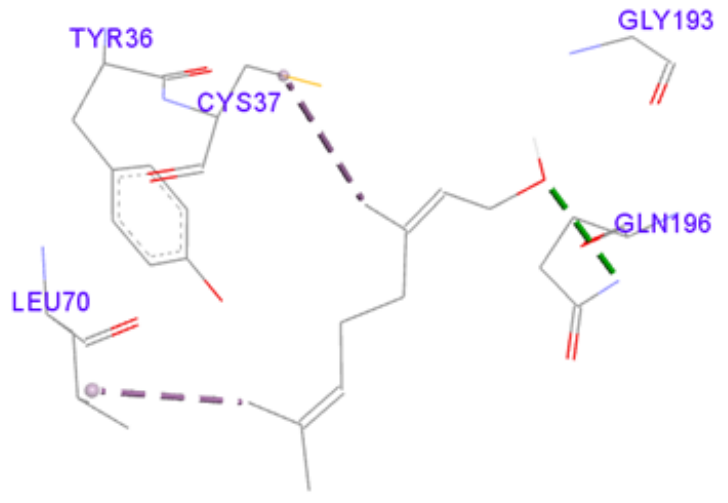

(A)

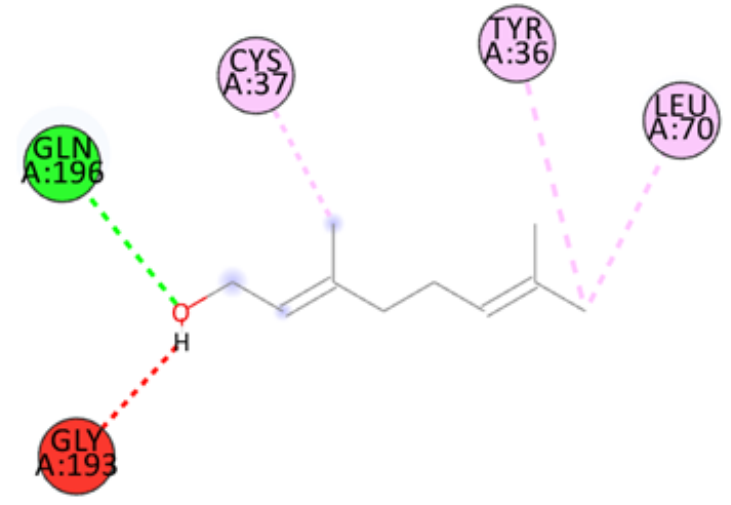

(B)

PDB ID: IJ_3,7-dimethyl-2,6-octadien-I-ol. 


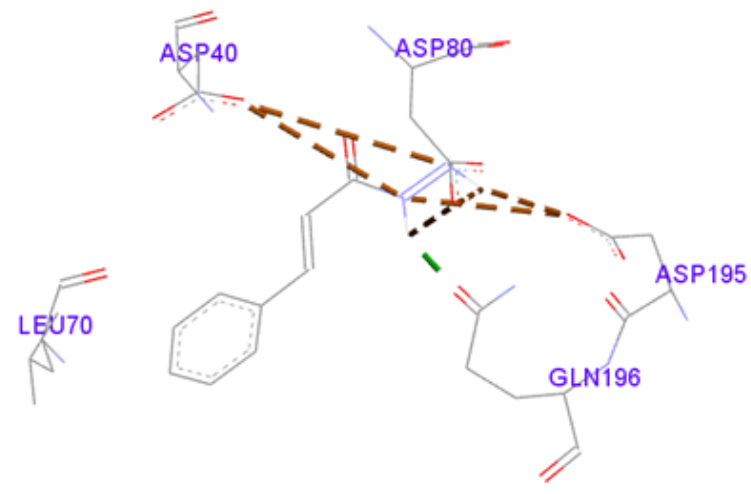

(A)

PDB ID: IJ_3-Phenyl-2-propenoic acid.

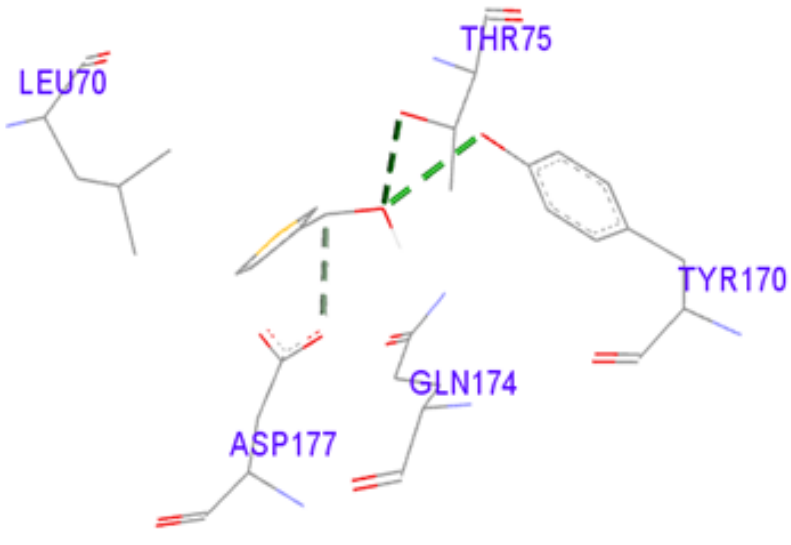

(A)
LEU

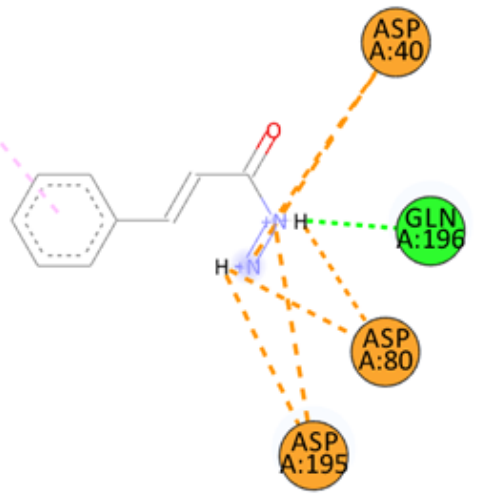

(B)

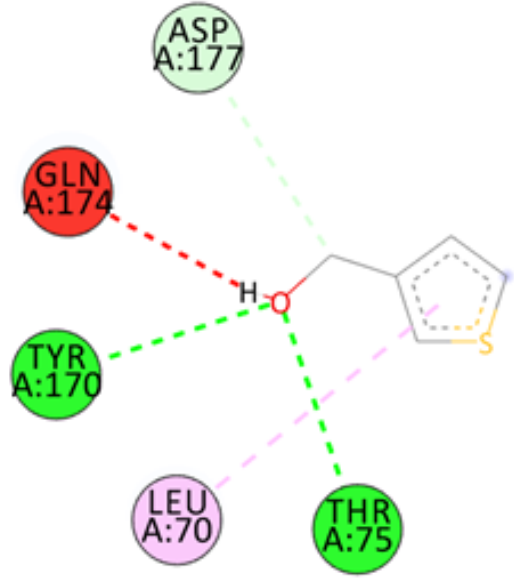

(B)

PDB ID: IJIJ3-Thiophenemethanol.

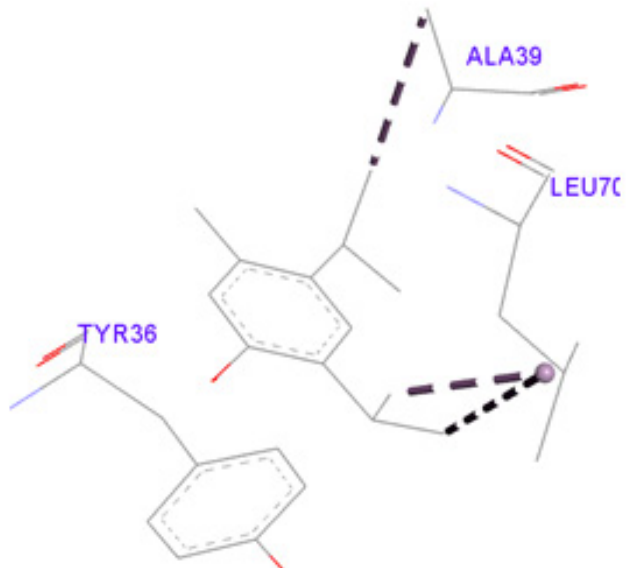

(A)

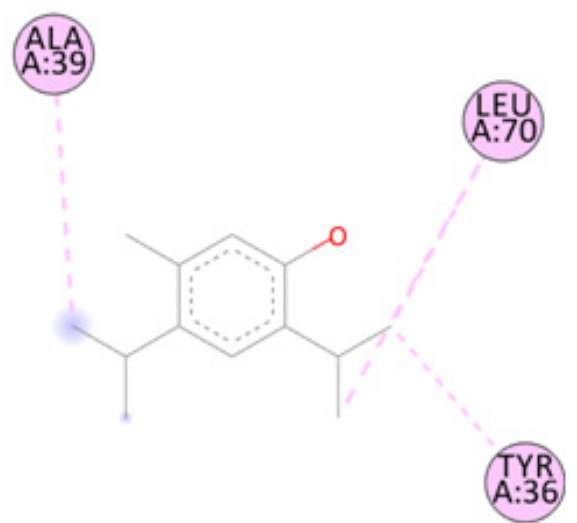

(B)

PDB ID: IJ_5-Methyl-2,4-diisopropylphenol. 


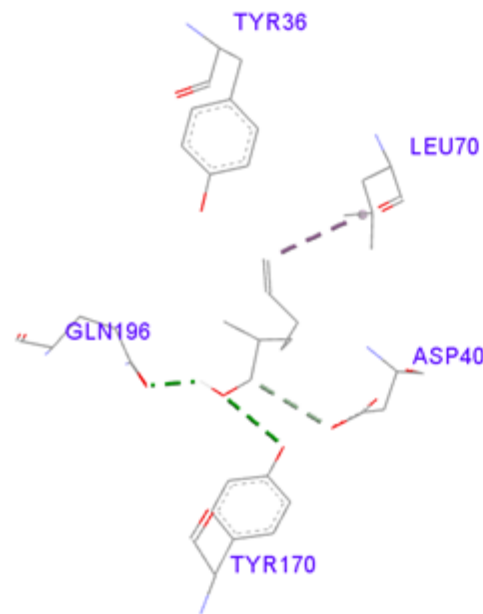

(A)

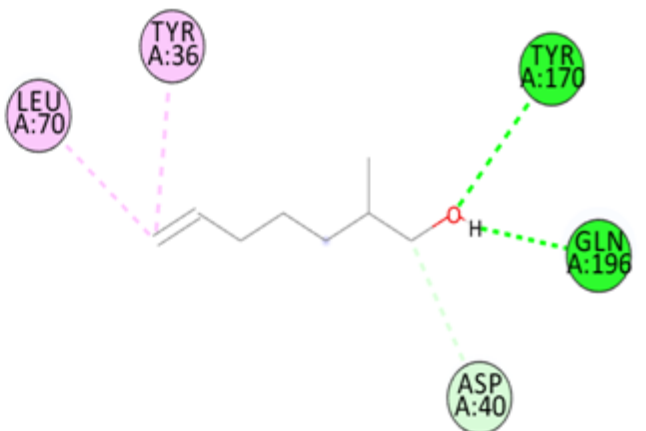

(B)

PDB ID: IJIJ6-hepten-I-ol, 2-Methyl.

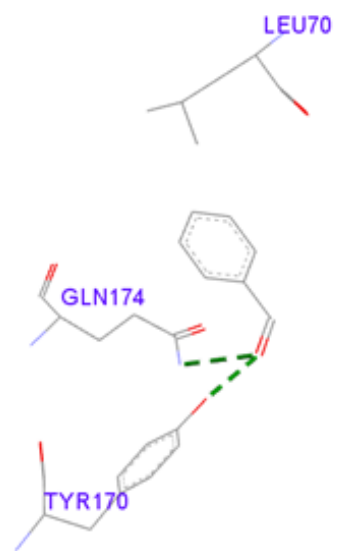

(A)

PDB ID: IJI_Benzaldehyde.

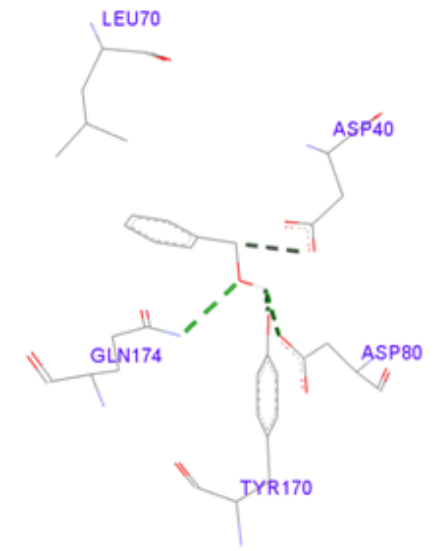

(A)

PDB ID: IJ]_Benzyl alcohol.

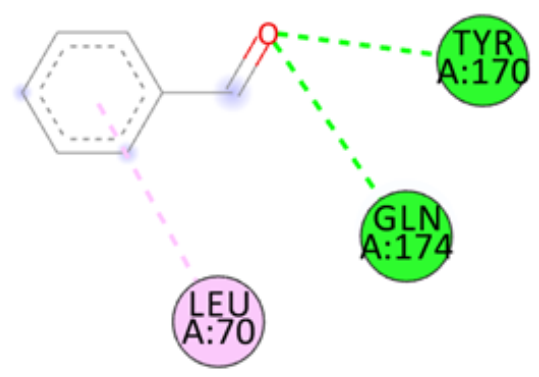

(B)

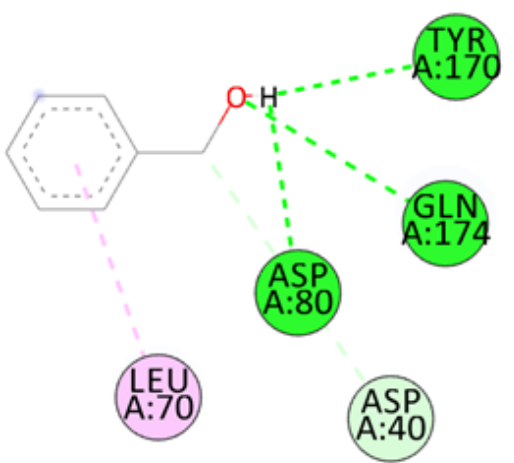

(B) 


$$
\text { LEU70 }
$$

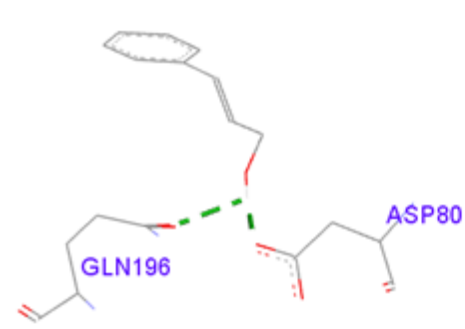

(A)

PDB ID: IJI_Cinnamyl alcohol.

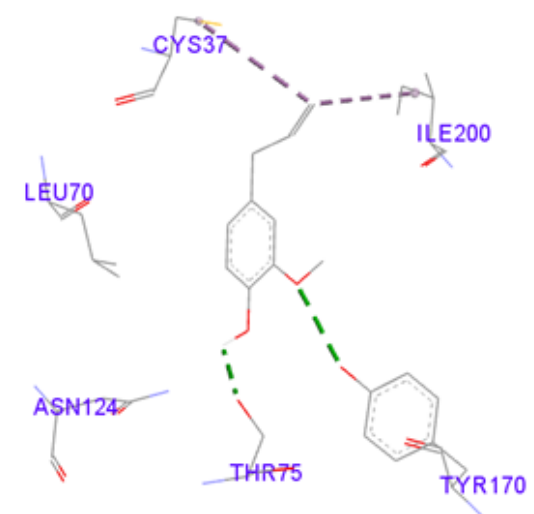

(A)

PDB ID: IJ_Eugenio.

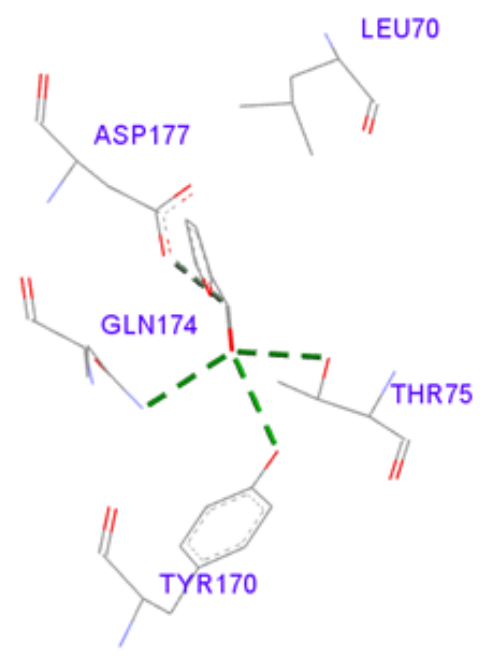

(A)

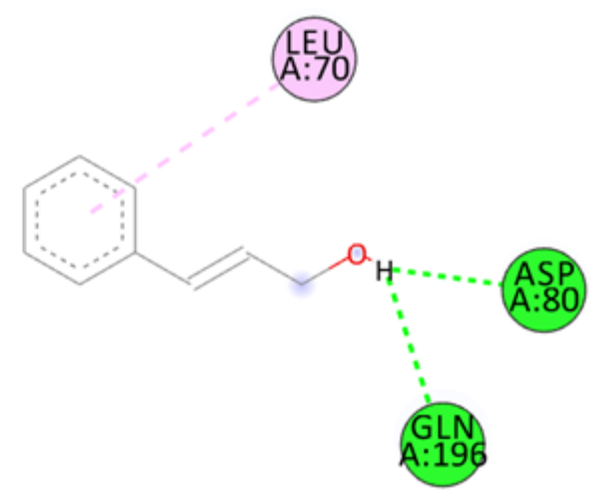

(B)

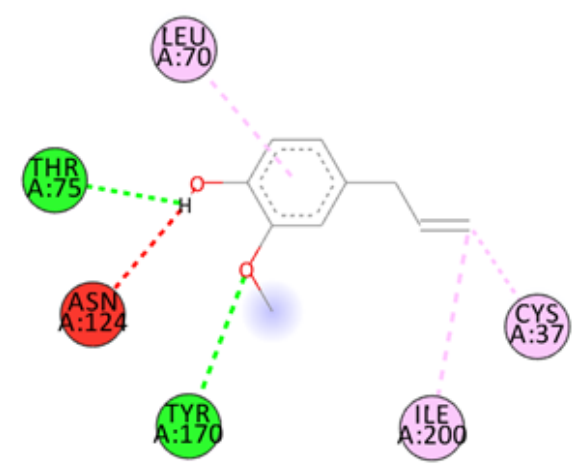

(B)

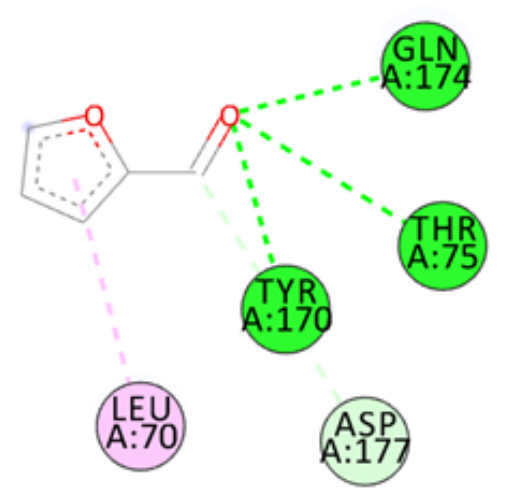

(B)

PDB ID: IJIJFurfural. 


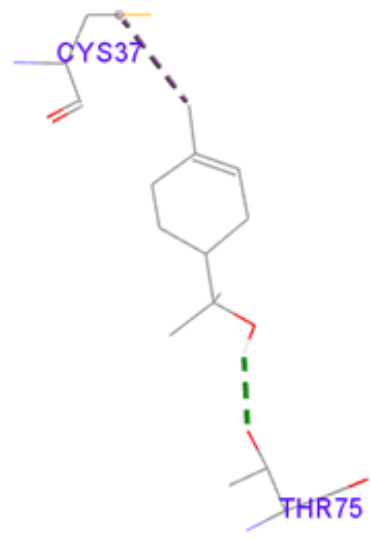

(A)

PDB ID: IJI_ $\alpha$-Terpineol.

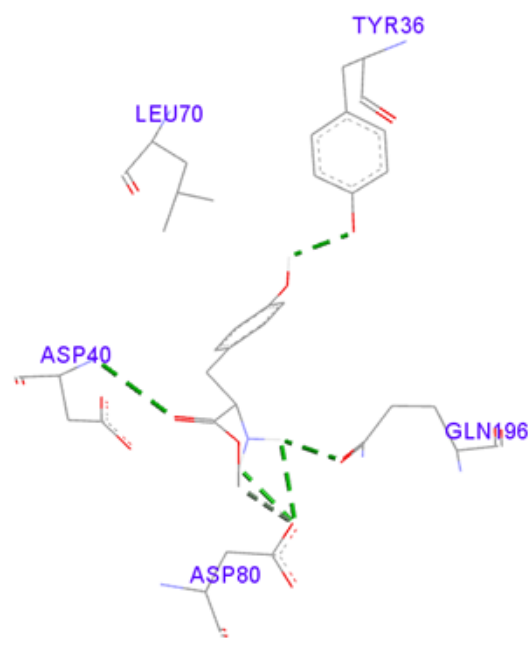

(A)

PDB ID: IJIJ_ L-Tyrosine Methyl ester.

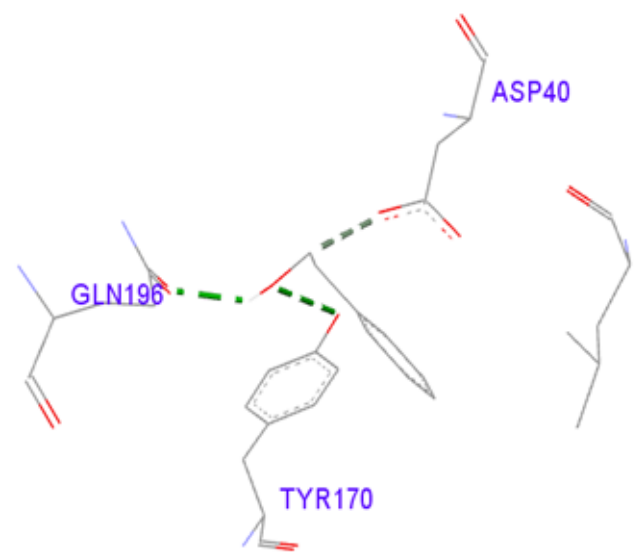

(A)

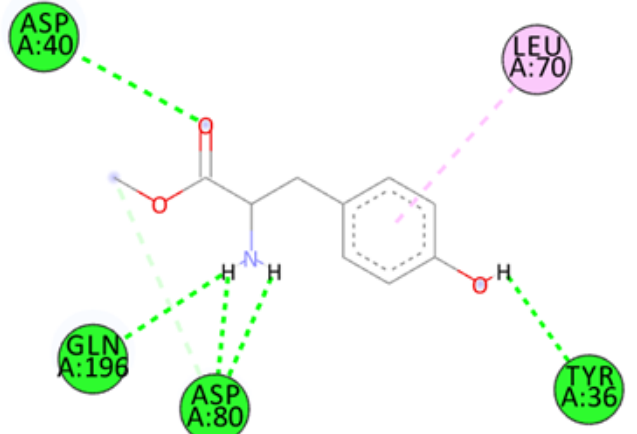

(B)

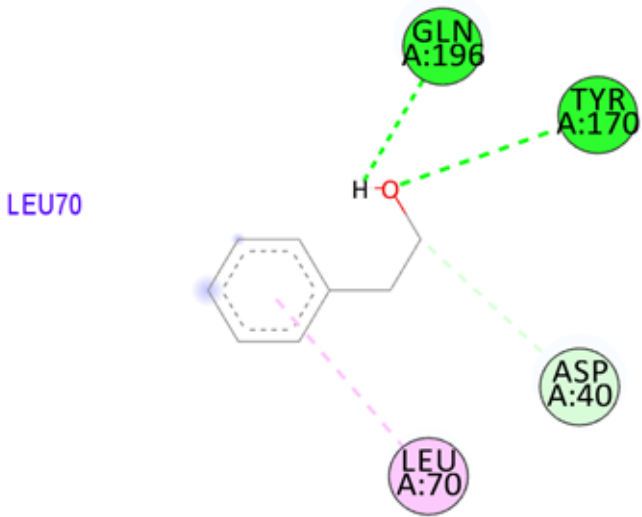

(B)

PDB ID: IJIJ_Phenethyl alcohol. 


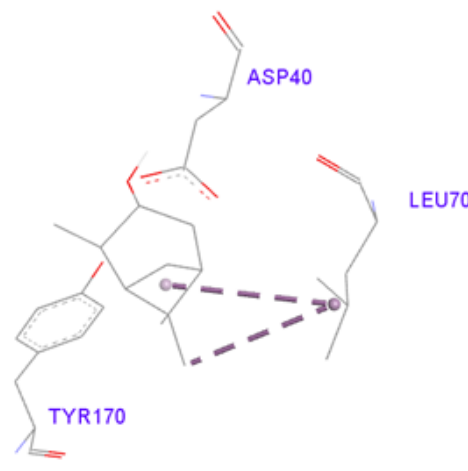

(A)

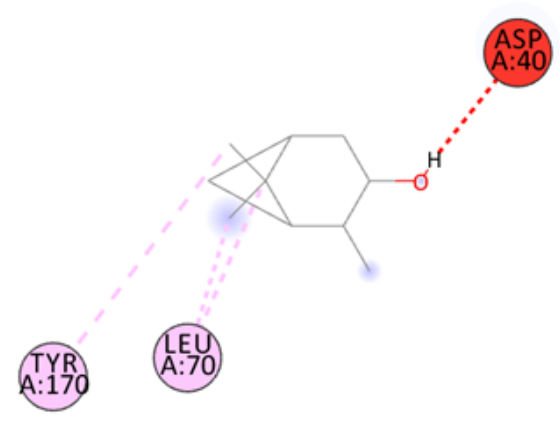

(B)

PDB ID: IJIJ_Pinocampheol.

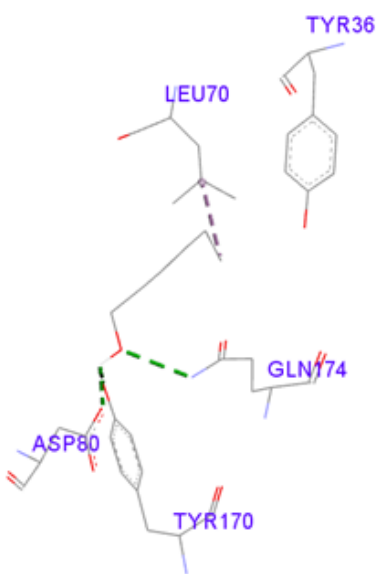

(A)

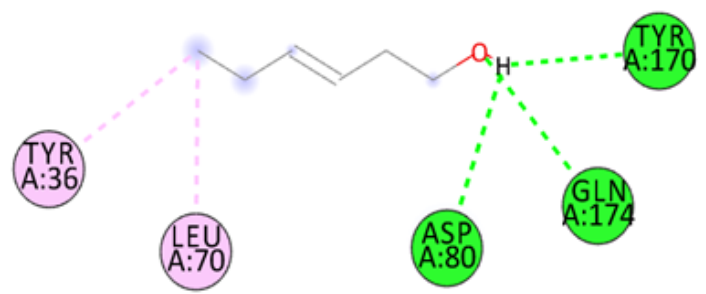

(B)

PDB ID: IJ]_Trans-3-Hexen-I-ol.

Figure 3 Docked pose of rigid ligand docking of Table I PDB ID: IJ (A) showing molecular interactions-hydrogen and hydrophobic bonds as green and pink/ purple dashed lines, respectively; (B) 2D plot of interactions between Ligand Receptor generated by BIOVIA.

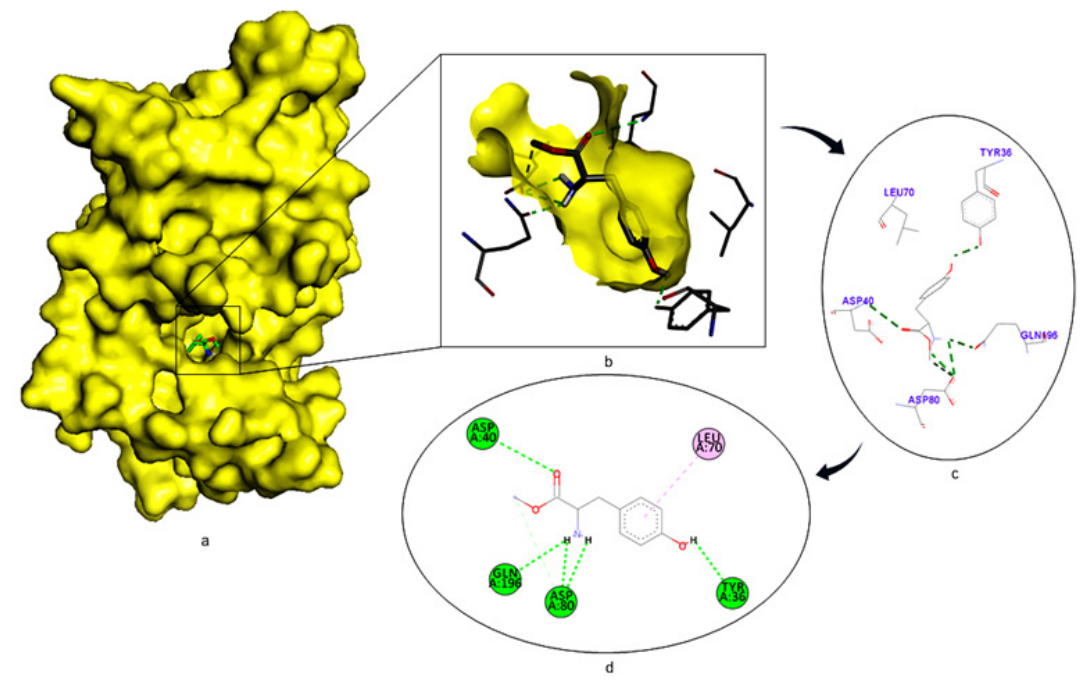

Figure 4 Interaction studies of S. aureus TyrRS against natural compounds (A) Surface representation of the complex file showing deep cavity (B) Zoom image in the interactions of active site residues complex file $(C)$ The interactions site showing the protein residues in S. aureus TyrRS protein. (D) Surface representation complex file showing the 2D interaction analysis done by Discovery studio (BIOVIA). 
Table 2 Docking score for the phytoconstituents of $C$. gigantea flower extract with PDB ID: IJJ.

\begin{tabular}{llll}
\hline S.No. & Compound & Formula & Docked score $(\mathrm{kcal} / \mathrm{mol})$ \\
\hline $\mathrm{I}$ & SB-239629 & - & -7.5 \\
2 & 2,3-Dihydro-benzofuran & $\mathrm{C}_{8} \mathrm{H}_{8} \mathrm{O}$ & -5.8 \\
3 & 2,4-dimethylacetophenone & $\mathrm{C}_{10} \mathrm{H}_{12} \mathrm{O}$ & -6.8 \\
4 & 2-Methoxy-4-vinylguaiacol & $\mathrm{C}_{10} \mathrm{H}_{14} \mathrm{O}_{3}$ & -6.5 \\
5 & 3,7-Dimethyl-I,6-octadien-3-ol & $\mathrm{C}_{10} \mathrm{H}_{18} \mathrm{O}$ & -5.2 \\
6 & 3,7-dimethyl-2,6-octadien-I-ol & $\mathrm{C}_{10} \mathrm{H}_{18} \mathrm{O}$ & -5.5 \\
7 & 3-Phenyl-2-propenoic_acid & $\mathrm{C}_{9} \mathrm{H}_{10} \mathrm{~N}_{2} \mathrm{O}$ & -6.6 \\
8 & 3-Thiophenemethanol & $\mathrm{C}_{5} \mathrm{H}_{6} \mathrm{OS}$ & -4.5 \\
9 & 5-Methyl-2,4-diisopropylphenol & $\mathrm{C}_{13} \mathrm{H}_{20} \mathrm{O}$ & -6.5 \\
10 & 6-hepten-I-ol, 2-Methyl & $\mathrm{C}_{8} \mathrm{H}_{16} \mathrm{O}$ & -4.9 \\
11 & Benzaldehyde & $\mathrm{C}_{7} \mathrm{H}_{6} \mathrm{O}$ & -5.2 \\
12 & Benzyl alcohol & $\mathrm{C}_{7} \mathrm{H}_{8} \mathrm{O}$ & -5.2 \\
13 & Cinnamyl alcohol & $\mathrm{C}_{9} \mathrm{H}_{10} \mathrm{O}$ & -5.7 \\
14 & Eugenol & $\mathrm{C}_{10} \mathrm{H}_{12} \mathrm{O}$ & -6.3 \\
15 & Furfural & $\mathrm{C}_{5} \mathrm{H}_{4} \mathrm{O}_{2}$ & -4.8 \\
16 & $\alpha$-Terpineol & $\mathrm{C}_{10} \mathrm{H}_{18} \mathrm{O}$ & -6.5 \\
17 & L-Tyrosine_Methyl_ester & $\mathrm{C}_{10} \mathrm{H}_{13} \mathrm{NO}$ & -6.7 \\
18 & Phenethyl alcohol & $\mathrm{C}_{8} \mathrm{H}_{10} \mathrm{O}$ & -5.6 \\
19 & Pinocampheol & $\mathrm{C}_{10} \mathrm{H}_{18} \mathrm{O}$ & -5.1 \\
20 & Trans-3-Hexen-I-ol & $\mathrm{C}_{6} \mathrm{H}_{12} \mathrm{O}$ & -4.5 \\
\hline
\end{tabular}

Table 3 Detailed molecular interactions obtained following the rigid ligand docking of PDB ID: IJ

\begin{tabular}{|c|c|c|c|c|}
\hline Ligand & Interacting residues & Distance (A) & Category & Type \\
\hline \multirow{10}{*}{ SB-239629 } & Tyr36 & 3.08 & H-Bond & Conventional \\
\hline & Cys 37 & 2.99 & H-Bond & Conventional \\
\hline & Gly38 & 2.20 & $\mathrm{H}$-Bond & Conventional \\
\hline & Asp40 & 3.07 & H-Bond & Conventional \\
\hline & Leu70 & 5.07 & Hydrophobic & Pi-Alkyl \\
\hline & Tyrl70 & 2.36 & H-Bond & Conventional \\
\hline & Gln 174 & 2.39 & Donor & Unfavorable \\
\hline & Aspl77 & 2.37 & $\mathrm{H}$-Bond & Conventional \\
\hline & Gly 193 & 2.63 & Donor & Unfavorable \\
\hline & Aspl95 & 2.23 & H-Bond & Conventional \\
\hline \multirow{3}{*}{ 2,3-Dihydro-benzofuran } & Leu70 & 4.91 & Hydrophobic & Pi-Alkyl \\
\hline & Tyr 170 & 2.87 & H-Bond & Conventional \\
\hline & Gln 174 & 3.20 & H-Bond & Conventional \\
\hline \multirow{7}{*}{ 2,4-dimethylacetophenone } & Tyr36 & 5.02 & & Pi-Alkyl \\
\hline & Cys 37 & 4.37 & Hydrophobic & Pi-Alkyl \\
\hline & Leu70 & 3.94 & $\begin{array}{l}\text { Hydrophobic } \\
\text { Hydrophobic }\end{array}$ & Pi-Alkyl \\
\hline & Thr75 & 3.13 & Hydrophobic & Conventional \\
\hline & Tyr 170 & 2.89 & $\mathrm{H}$-Bond & Conventional \\
\hline & $\mathrm{G} \ln 174$ & 3.29 & H-Bond & Conventional \\
\hline & Ile200 & 5.35 & H-Bond Hydrophobic & Pi-Alkyl \\
\hline \multirow{6}{*}{ 2-Methoxy-4-vinylguaiacol } & Cys37 & 4.39 & Hydrophobic & Pi-Alkyl \\
\hline & Gly38 & 2.61 & $\mathrm{CH}$-Bond & Conventional \\
\hline & Asp80 & 3.51 & H-Bond & Conventional \\
\hline & Tyrl70 & 2.89 & H-Bond & Conventional \\
\hline & Gln 174 & 3.08 & $\mathrm{H}$-Bond & Conventional \\
\hline & Aspl77 & 3.25 & $\mathrm{CH}$-Bond & Conventional \\
\hline
\end{tabular}


Table continue

\begin{tabular}{|c|c|c|c|c|}
\hline Ligand & Interacting residues & Distance $(\AA)$ & Category & Type \\
\hline \multirow{5}{*}{$\begin{array}{l}\text { 3,7-Dimethyl-I,6-octadien- } \\
\text { 3-ol }\end{array}$} & Tyr36 & 4.98 & Hydrophobic & Pi-Alkyl \\
\hline & Leu70 & 4.00 & Hydrophobic & Pi-Alkyl \\
\hline & Tyr I70 & 3.24 & H-Bond & Conventional \\
\hline & Gln 174 & 2.65 & Donor & Unfavorable \\
\hline & $G \ln 196$ & 2.20 & H-Bond & Conventional \\
\hline \multirow{5}{*}{$\begin{array}{l}\text { 3,7-dimethyl-2,6-octadien- } \\
\text { I-ol }\end{array}$} & Tyr36 & 5.25 & Hydrophobic & Pi-Alkyl \\
\hline & Cys37 & 4.21 & Hydrophobic & Pi-Alkyl \\
\hline & Leu70 & 4.01 & Hydrophobic & Pi-Alkyl \\
\hline & Gly193 & 2.30 & Donor & Unfavorable \\
\hline & Gln 196 & 3.12 & H-Bond & Conventional \\
\hline \multirow{5}{*}{ 3-Phenyl-2-propenoic_acid } & Asp40 & 4.72 & H-Bond & Salt Bridge \\
\hline & Asp80 & 2.66 & $\mathrm{H}$-Bond & Salt Bridge \\
\hline & Leu70 & 4.83 & Hydrophobic & Pi-Alkyl \\
\hline & Aspl95 & 3.12 & H-Bond & Salt Bridge \\
\hline & Gln 196 & 1.91 & H-Bond & Conventional \\
\hline \multirow{5}{*}{ 3-Thiophenemethanol } & Leu70 & 5.30 & Hydrophobic & Pi-Alkyl \\
\hline & Thr75 & 2.97 & H-Bond & Conventional \\
\hline & Tyr I70 & 2.97 & Donor & Unfavorable \\
\hline & Gln I74 & 2.66 & $\mathrm{H}$-Bond & Conventional \\
\hline & Aspl77 & 3.52 & $\mathrm{CH}$-Bond & Conventional \\
\hline \multirow{3}{*}{$\begin{array}{l}\text { 5-Methyl-2,4- } \\
\text { diisopropylphenol }\end{array}$} & Tyr36 & 4.52 & Hydrophobic & Pi-Alkyl \\
\hline & Ala39 & 4.25 & Hydrophobic & Pi-Alkyl \\
\hline & Leu70 & 3.56 & Hydrophobic & Pi-Alkyl \\
\hline \multirow{5}{*}{ 6-hepten-I-ol,2-Methyl } & Tyr36 & 4.78 & Hydrophobic & Pi-Alkyl \\
\hline & Asp40 & 3.42 & $\mathrm{CH}$-Bond & Conventional \\
\hline & Leu70 & 3.86 & Hydrophobic & Pi-Alkyl \\
\hline & Tyr I70 & 3.17 & H-Bond & Conventional \\
\hline & Gln 196 & 2.09 & H-Bond & Conventional \\
\hline \multirow{3}{*}{ Benzaldehyde } & Leu70 & 4.88 & Hydrophobic & Pi-Alkyl \\
\hline & Tyr I70 & 2.94 & H-Bond & Conventional \\
\hline & Gln 174 & 2.94 & $\mathrm{H}$-Bond & Conventional \\
\hline \multirow{5}{*}{ Benzyl alcohol } & Asp40 & 3.70 & C H-Bond & Conventional \\
\hline & Leu70 & 5.24 & Hydrophobic & Pi-Alkyl \\
\hline & Asp80 & 2.80 & H-Bond & Conventional \\
\hline & Tyrl70 & 2.63 & H-Bond & Conventional \\
\hline & Gln 174 & 2.91 & H-Bond & Conventional \\
\hline \multirow{3}{*}{ Cinnamyl alcohol } & Leu70 & 5.18 & Hydrophobic & Pi-Alkyl \\
\hline & Asp80 & 1.97 & H-Bond & Conventional \\
\hline & Gln 196 & 2.40 & $\mathrm{H}$-Bond & Conventional \\
\hline \multirow{6}{*}{ Eugenol } & Cys37 & 4.65 & Hydrophobic & \\
\hline & Leu70 & 4.79 & Hydrophobic & Pi-Alkyl \\
\hline & Thr75 & 2.23 & H-Bond & Pi-Alkyl \\
\hline & Asn I 24 & 2.49 & Donor & Conventional Unfavorable \\
\hline & Tyr I70 & 2.99 & H-Bond & Conventional Pi-Alkyl \\
\hline & Ile200 & 5.27 & Hydrophobic & \\
\hline \multirow{5}{*}{ Furfural } & Leu70 & 4.86 & Hydrophobic & Pi-Alkyl \\
\hline & Thr75 & 2.98 & H-Bond & Conventional \\
\hline & Tyr I70 & 2.92 & H-Bond & Conventional \\
\hline & Gln I74 & 2.98 & H-Bond & Conventional \\
\hline & Aspl77 & 3.34 & $\mathrm{CH}$-Bond & Conventional \\
\hline \multirow{2}{*}{$\alpha$-Terpineol } & Cys37 & 4.48 & Hydrophobic & Pi-Alkyl \\
\hline & Thr75 & 2.82 & $\mathrm{H}$-Bond & Conventional \\
\hline \multirow{5}{*}{ L-Tyrosine_Methyl_ester } & Tyr36 & 2.30 & H-Bond & \\
\hline & Asp40 & 3.24 & Hydrophobic & Conventional PI-Alkyl \\
\hline & Leu70 & 5.30 & H-Bond & Conventional \\
\hline & Asp80 & 2.51 & $\mathrm{H}$-Bond & Conventional \\
\hline & $G \ln 196$ & 2.35 & $\mathrm{H}$-Bond & Conventional \\
\hline
\end{tabular}


Table continue

\begin{tabular}{|c|c|c|c|c|}
\hline Ligand & Interacting residues & Distance (A) & Category & Type \\
\hline Phenethyl alcohol & $\begin{array}{l}\text { Asp40 } \\
\text { Leu70 } \\
\text { Tyr I70 } \\
\text { GIn I96 }\end{array}$ & $\begin{array}{l}3.63 \\
4.87 \\
3.13 \\
2.16\end{array}$ & $\begin{array}{l}\text { C H-Bond } \\
\text { Hydrophobic } \\
\text { H-Bond } \\
\text { H-Bond }\end{array}$ & $\begin{array}{l}\text { Conventional Pi-Alkyl } \\
\text { Conventional } \\
\text { Conventional }\end{array}$ \\
\hline Pinocampheol & $\begin{array}{l}\text { Asp40 } \\
\text { Leu70 } \\
\text { Tyr I70 }\end{array}$ & $\begin{array}{l}2.16 \\
4.39 \\
5.47\end{array}$ & $\begin{array}{l}\text { Donor } \\
\text { Hydrophobic } \\
\text { Hydrophobic }\end{array}$ & $\begin{array}{l}\text { Unfavorable } \\
\text { Pi-Alkyl } \\
\text { Pi-Alkyl }\end{array}$ \\
\hline Trans-3-Hexen-I-ol & $\begin{array}{l}\text { Tyr36 } \\
\text { Leu70 } \\
\text { Asp80 } \\
\text { Tyr I70 } \\
\text { GInI74 }\end{array}$ & $\begin{array}{l}5.26 \\
4.06 \\
2.68 \\
2.53 \\
2.88\end{array}$ & $\begin{array}{l}\text { Hydrophobic } \\
\text { Hydrophobic } \\
\text { H-Bond } \\
\text { H-Bond } \\
\text { H-Bond }\end{array}$ & $\begin{array}{l}\text { Pi-Alkyl } \\
\text { Pi-Alkyl } \\
\text { Conventional } \\
\text { Conventional } \\
\text { Conventional }\end{array}$ \\
\hline
\end{tabular}

\section{Discussion}

C. gigantea flower extract naturals compounds, the physiochemical analysis and RO5 analysis shows that all those compound having $>500$ MW, $>10$ number of rotatable bonds (RB), hydrogen bond donor and acceptor $>5$ bonds and $\log \mathrm{P}$ value is $>3$ all the properties showing these compounds are good for bioavailability of the drug. ${ }^{6-8,14,15}$ The predicted ligand binding site for $S$. aureus TyrRS protein showing

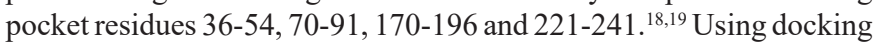
analysis, the natural compounds against $S$. aureus TyrRS having top 5 docking scores compounds are 2,4-dimethylacetophenone (-6.8), L-Tyrosine_Methyl_ester (-6.7), 3-Phenyl-2-propenoic_acid (-6.6), 2-Methoxy-4-vinylguaiacol (-6.5) and $\alpha$-Terpineol (-6.5). ${ }^{20,21,23}$ Protein-ligands interaction studies of $S$. aureus TyrRS ternary complexes with natural compounds as the putative inhibitor. In complex file interaction studies showing all these natural compounds closely interacted with active sites /substrate binding domains. The interaction residues of the ternary structure of $S$. aureus TyrRS protein are Tyr36, Cys37, Gly38, Ala39, As40, Leu70, Asp80, Thr75, Tyr170, Gln174, Asp177, Gly193, Asp195, Gln196, Ile 200. This interaction study confirmed that there was the involvement of Hydrogen bond (H-bond), Carbon-Hydrogen bond (C-H bond) and Pi-Alkyl bond. The interaction study showing the strong interaction between $S$. aureus TyrRS protein with natural compounds but the L-Tyrosine Methyl ester docking showing the maximum four hydrogen bond interaction with TYR36, ASP40, ASP80 and GLN19 residues and al the residues are closely interacted with active sites. ${ }^{26-29}$ The outcome was determined that the phytocompounds of $C$. gigantea can be used as a natural better therapeutic target for antimicrobial resource and therefore targeting natural compounds might be an advantageous step in the direction of therapeutic development.

\section{Conclusions}

A C. gigantea flower part with auxiliary highlights of flavonoids content was read for their antibacterial and cancer prevention agent profiles. The $C$. gigantea flower progressive has most noteworthy antibacterial action against an ESKAPE pathogenic strains of Gramnegative and Gram-positive microbes, including a MRSA strain, just as a fascinating cancer prevention agent profile. The occurrence of a phytoconstituents could be significant as it is an auxiliary component of the most dynamic substances. These substances or compounds could be considered for its antibacterial potential and could be a significant hotspot for the plan and advancement of new foreign agent of infective material. As the most dynamic compound for additional examinations, docking concentrates with significant objective were performed to explain a possible system of activity for this compound.
PDB ID:1JIJ, tyrosyl-tRNA synthetase from $S$. aureus were studied as potential targets, and a correlation between the observed inhibitory activity and the in silico molecular docking scores was obtained. Moreover, compounds also approved by RO5 drug likeness properties. Future studies will be carried out to evaluate the anti-biofilm activity of this compound and to explore the biological activities of the metal complexes to find new compounds with increased antimicrobial potential and a broader spectrum activity.

\section{Acknowledgements}

The authors acknowledge support from the Centre for Interdisciplinary Research in Basic Science, Jamia Millia Islamia University. Md. Amjad Beg also acknowledges University Grants Commission Maulana Azad National Fellowship for the financial support and Jamia Millia Islamia University.

\section{Conflicts of Interest}

The authors declare that they have no potential conflict of interests.

\section{References}

1. Mata R, Figueroa M, Navarrete A, et al. Chemistry and Biology of Selected Mexican Medicinal Plants. Prog Chem Org Nat Prod. 2019;108:1-142.

2. Cowan MM. Plant products as antimicrobial agents. Clin Microbiol Rev. 1999;12(4):564-582.

3. Ahvazi M, Khalighi-Sigaroodi F, Charkhchiyan MM, et al. Introduction of medicinal plants species with the most traditional usage in alamut region. Iran J Pharm Res. 2012;11(1):185-194.

4. Shami AM, Philip K, Muniandy S. Synergy of antibacterial and antioxidant activities from crude extracts and peptides of selected plant mixture. BMC Complement Altern Med. 2013;13:360.

5. Vadnere GP, Gaud RS, Singhai, AK, et al. Effect of Calotropis gigantea flowers extracts on mast cell degranulation in rats. Pharmacologyonline. 2010;3:298-303.

6. Beg MA, Athar F. Pharmacokinetic and molecular docking studies of Achyranthes aspera phytocompounds to exploring potential antituberculosis activity. J Bacteriol Mycol Open Access. 2020;8(1):18-27.

7. Minakshi S, Kalim J. Comparative study of chemical composition of Calotropis gigantea flower, leaf and fruit essential oil. Eur Chem Bull. 2015;4(10):477-480.

8. Kumar G, Loganathan K, Rao B. A review on pharmacological and phytochemical profile of Calotropis gigantea Linn. Pharmacologyonline. $2011 ; 1: 1-8$ 
9. Chambers HF, Deleo FR. Waves of resistance: Staphylococcus aureus in the antibiotic era. Nat Rev Microbiol. 2009;7(9):629-641.

10. Elsner HA, Sobottka I, Mack D, et al. Virulence factors of Enterococcus faecalis and Enterococcus faecium blood culture isolates. Eur J Clin Microbiol Infect Dis. 2000;19(1):39-42.

11. Houang ET, Sormunen RT, Lai L, et al. Effect of desiccation on the ultrastructural appearances of Acinetobacter baumannii and Acinetobacter lwoffii. J Clin Pathol. 1998;51(10):786-788.

12. Høiby N, Bjarnsholt T, Givskov M, et al. Antibiotic resistance of bacterial biofilms. Int J Antimicrob Agents. 2010;35(4):322-332.

13. Kumarasamy KK, Toleman MA, Walsh TR, et al. Emergence of a new antibiotic resistance mechanism in India, Pakistan, and the UK a molecular, biological, and epidemiological study. Lancet Infect Dis. 2010;10(9):597-602.

14. Gurib-Fakim A. Medicinal plants: traditions of yesterday and drugs of tomorrow. Mol Aspects Med. 2006;27(1):1-93.

15. Daina A, Michielin O, Zoete V. SwissADME: a free web tool to evaluate pharmacokinetics, drug-likeness and medicinal chemistry friendliness of small molecules. Sci Rep. 2017;7:42717.

16. Beg MA, Thakur SC, Athar F. Molecular modeling and in silico characterization of mycobacterial Rv3101c and Rv3102c proteins: prerequisite molecular target in cell division. Pharm Pharmacol Int J. 2020;8(4):234-243

17. Beg MA, Thakur SC, Athar F. Computational annotations of mycobacterial Rv3632 that confers its efficient function in cell wall biogenesis. J Bacteriol Mycol Open Access. 2020;8(2):46-53.

18. Qiu X, Janson CA, Smith WW, et al. Crystal structure of Staphylococcus aureus tyrosyl-tRNA synthetase in complex with a class of potent and specific inhibitors. Protein Sci. 2001;10(10):2008-2016.

19. Tian W, Chen C, Lei X, et al. CASTp 3.0: computed atlas of surface topography of proteins. Nucleic Acids Res. 2018;46(W1):W363-W367.
20. Beg MA, Athar F. Anti-HIV and Anti-HCV drugs are the putative inhibitors of RNA-dependent-RNA polymerase activity of NSP12 of the SARS CoV-2 (COVID-19). Pharm Pharmacol Int J. 2020;8(3):163-172.

21. Trott O, Olson AJ. AutoDock Vina: improving the speed and accuracy of docking with a new scoring function, efficient optimization, and multithreading. J Comput Chem. 2010;31(2):455-461.

22. Beg MA, Athar F. Computational method in COVID-19: Revelation of Preliminary mutations of RdRp of SARS CoV-2 that build new horizons for therapeutic development. J Hum Virol Retrovirolog. 2020;8(3):62-72.

23. Forli S, Huey R, Pique ME, et al. Computational protein-ligand docking and virtual drug screening with the AutoDock suite. Nat Protoc. 2016;11(5):905-919.

24. Beg MA, Shivangi, Thakur SC, et al. Systematical Analysis to Assist the Significance of Rv1907c Gene with the Pathogenic Potentials of Mycobacterium tuberculosis H37Rv. J Biotechnol Biomaterial. 2019;8:287.

25. Shivangi, Beg MA, Meena LS. Insights of Rv2921c (ftsY) gene of Mycobacterium tuberculosis H37Rv to prove its significance by computational means. Biomed J Sci \& Tech Res. 2018;12(2).

26. Rigsby RE, Parker AB. Using the PyMOL application to reinforce visual understanding of protein structure. Biochem Mol Biol Educ. 2016;44(5):433-437.

27. Biovia DS. Discovery studio modeling environment. San Diego: Dassault Systems. 2015.

28. Beg MA, Shivangi, Thakur SC, et al. Structural Prediction and Mutational Analysis of Rv3906c Gene of Mycobacterium tuberculosis H37Rv to Determine Its Essentiality in Survival. Adv Bioinformatics. 2018;2018:6152014

29. Beg MA, Shivangi, Fareeda A, et al. Structural And Functional Annotation Of Rv1514c Gene Of Mycobacterium tuberculosis H37Rv As Glycosyl Transferases. J Adv Res Biotech. 2018;3(2):1-9. 